\title{
Posterior parietal cortex dynamically ranks topographic signals via cholinergic influence
}

\author{
John I. Broussard* \\ Department of Neuroscience, Center on Addiction, Learning, Memory, Baylor College of Medicine, Houston, TX, USA
}

\section{Edited by:}

David J. Bucci, Dartmouth College USA

\section{Reviewed by:}

David J. Bucci, Dartmouth College, USA

Christiane Thiel, Carl von Ossietzky Universität Oldenburg, Germany

\section{*Correspondence:}

John Broussard, Department of Neuroscience, Center on Addiction, Learning, Memory, Baylor College of Medicine, One Baylor Plaza, Houston, TX 77030, USA. e-mail: johnbroussard.phd@ gmail.com
The hypothesis to be discussed in this review is that posterior parietal cortex (PPC) is directly involved in selecting relevant stimuli and filtering irrelevant distractors. The PPC receives input from several sensory modalities and integrates them in part to direct the allocation of resources to optimize gains. In conjunction with prefrontal cortex, nucleus accumbens, and basal forebrain cholinergic nuclei, it comprises a network mediating sustained attentional performance. Numerous anatomical, neurophysiological, and lesion studies have substantiated the notion that the basic functions of the PPC are conserved from rodents to humans. One such function is the detection and selection of relevant stimuli necessary for making optimal choices or responses. The issues to be addressed here are how behaviorally relevant targets recruit oscillatory potentials and spiking activity of posterior parietal neurons compared to similar yet irrelevant stimuli. Further, the influence of cortical cholinergic input to PPC in learning and decision-making is also discussed. I propose that these neurophysiological correlates of attention are transmitted to frontal cortical areas contributing to the top-down selection of stimuli in a timely manner.

Keywords: sustained attention, acetylcholine, norepinephrine, prefrontal cortex, posterior parietal cortex, P300, contingent negative variation, muscarinic

\section{INTRODUCTION: PARIETAL COMPONENT OF A NEURONAL CIRCUIT MEDIATING VIGILANCE}

As part of the dorsal stream of visual processing, one of the hallmark functions the posterior parietal cortex (PPC) is the orientation of a subject to novel or meaningful stimuli. Critchley exhaustively documented that patients with unilateral damage to the PPC demonstrate neglect, a clinical syndrome characterized by an inability to report to stimuli presented on the contralateral side (Critchley, 1953). Posner and colleagues integrated data from single neuron recordings in monkeys, imaging studies and the study of patients with parietal lobe injuries to propose a theory that neglect results from an impaired ability to disengage attention from non-neglected side of space (Posner and Raichle, 1994). In rodents unilateral lesions in an anatomical homolog of the PPC produced persistent contralesional neglect to visual, auditory, and tactile stimuli, and disorders of spatial processing (King and Corwin, 1993; Reep and Corwin, 2009).

A series of neurophysiological studies in non-human primates provide evidence indicating that PPC neural activity represents the intentions of a subject to move in space, and that the PPC acts to guide effectors such as hands and eyes throughout space (Mountcastle et al., 1975; Kalaska, 1996; Snyder, 2000; Andersen and Buneo, 2002; Scherberger et al., 2005). Contrasting evidence indicates that PPC activity correlates with covert shifts in attention in the absence of effector movement (Colby and Goldberg, 1999; Bisley et al., 2004; Bisley and Goldberg, 2006; Ipata et al., 2006). An integration of the two neurophysiological models of parietal function complements the observations found in clinical research, namely that loss of parietal function impairs attention to and moving through contralateral space (Rushworth and Taylor, 2006). Taken together, the role of the PPC may then be to bias the detection and selection of sensory inputs from multiple modalities and to project target information to motor areas (Posner et al., 1980; Kastner and Ungerleider, 2000) ${ }^{1}$.

This review proposes the hypothesis that the PPC ranks and highlights behaviorally relevant stimuli in order to aid detection and guide navigation. The neural circuitry influencing parietal processing is described, including the prefrontal cortex and the neuromodulatory influence of the basal forebrain cholinergic system (BFCS) to PPC, and this review makes the case that this circuitry is necessary for continually updating the ranking of topological stimuli, such as in the presence of task-irrelevant stimuli or rule changes requiring new learning. The possible contribution of ascending noradrenergic (NE) projections is also discussed. Although there is less direct evidence of the NE influence over parietal processing in attention, NE has an effect on evoked responses measured from sensory cortices. Further, recordings from ascending NE projections indicate that in conditions requiring global enhancement of arousal these inputs may facilitate processing of thalamocortical signals.

\footnotetext{
${ }^{1}$ I use the term "detection" to describe a cognitive process consisting of "...the entry of information concerning the presence of a signal into a system that allows the subject to report the existence of the signal by an arbitrary response indicated by the experimenter" (15).
} 


\section{THE POSTERIOR PARIETAL CORTEX IN RODENT MODELS OF ATTENTION \\ IDENTIFICATION OF THE ANATOMICAL HOMOLOG OF RODENT POSTERIOR PARIETAL CORTEX}

Anatomically, the PPC of primates includes the superior and inferior parietal lobules. The superior lobules are comprised of Brodmann Areas (BA) 7 and 5, and the inferior lobules are comprised of BA 39 and 40. In monkeys and humans an intraparietal sulcus (IPS) delineates the superior and inferior lobules, and in monkeys neuronal activity within the lateral IPS corresponds to representations of salient stimuli in allocentric space (Colby and Goldberg, 1999), whereas activity in the medial IPS corresponds to the intention of a subject to reach for a target (Cohen and Andersen, 2002). The PPC expanded markedly in humans relative to monkeys, and evidence for homologous structures between the two is far from clear, though evidence for a human homolog of macaque LIP (Sereno et al., 2001), and motion sensitive activations were found in the ventral intraparietal sulcus (VIPS) and the VIP of macaques (Vanduffel et al., 2001, 2002).

In rats, the parcellation of PPC is not as precise. Anatomical features of the PPC include an interconnection to aspects of the visual system, including the frontal eye fields, pulvinar, ventrolateral thalamic nuclei (Leichnetz, 2001), and superior colliculus (Pare and Wurtz, 1997). In rats, the region considered to be a homolog to the primate PPC is generally defined as a region $3.5-5.0 \mathrm{~mm}$ caudal to the bregma and extending $1.5-5.0 \mathrm{~mm}$ lateral from the midline (Reep et al., 1994; Reep and Corwin, 2009). Rat PPC has reciprocal connections with the lateral dorsal and lateral posterior thalamic nuclei, similar to that of primates (Chandler et al., 1992). The PPC also has connections with medial agranular and orbital cortex, and is connected to auditory, somatosensory, and visual cortical areas (Reep et al., 1994). Further, basal forebrain cholinergic neurons project to the PPC of rats (Bucci et al., 1999). Although the distinctions of function (i.e., the parietal reach region vs. visual salience maps of the LIP) between subfields of the PPC have not been delineated in rodents, observations from the anatomical studies listed above support the general hypothesis that rat PPC is homologous to primate PPC, and is important for integrating multiple modes of sensory input for attentional processing.

\section{PARIETAL CORTEX DYNAMICALLY RANKS RELEVANT SIGNALS IN VISUAL ATTENTION TASKS}

There are two commonly used tasks to assess visual attention in rodents, the first being the 5 choice serial reaction task (5CSRTT) that was modeled after human continuous performance tasks (Carli et al., 1983; Bari et al., 2008), In the 5CSRTT, food-deprived rodents must monitor a horizontal array of five lights for brief, unpredictable flashes, and respond by nosepoking into the hole that flashed the light. The spatial position of the light varies on each trial and each correctly detected signal is rewarded with a food pellet. The second is the sustained attention task (SAT) developed by Bushnell and colleagues (Bushnell et al., 1994) and modified for visual attention by McGaughy and Sarter (1995). In the SAT, food- or water- deprived visual signals and blank trials are randomly presented. Responses are either hit or miss on cued trials, and correct rejection or false alarms on blank trials. Correct responses (hit or correct rejections) are rewarded and incorrect responses (misses or false alarms) initiate an intertrial interval without other consequences. In the SAT task, visual distractors can also be introduced to provide more challenging conditions (dSAT), and these characteristically impair detection of visual signals (Gill et al., 2000).

Rats in the dSAT first participate in a block of undistracted trials (about 50) before the distractors are presented. The distractor flashes at $0.5 \mathrm{~Hz}$ for $12 \mathrm{~min}$, meaning that the 25 unpredictable signals are diluted among 360 false signals. Even after familiarization with the dSAT rats have elevated false alarms. Here increased false alarms can be reconceptualized as a prediction error as the predicted outcome of a reward is different than the actual outcome of no reward (Schultz and Dickinson, 2000). Following several false alarms, rats improve performance but are still relatively impaired. Thus, observations indicate that the rodent learns that reporting salient yet irrelevant light signals is a failing strategy, and then begin to actively filter out subsequent distractor flashes.

Loss of BFCS input to the cortex impairs performance in both the 5CSRTT (Muir et al., 1994) and the SAT (McGaughy et al., 1996; Bushnell et al., 1998; Chiba et al., 1999). Due to the role of PPC in visual attention in primates and the innervation of BFCS input to this region, parietal cholinergic deafferentation was attempted in both of these paradigms. Cholinergic deafferentation in the PPC did not produce any deficits in the standard version of the 5CSRTT (Maddux et al., 2007). However, in a Pavlovian overshadowing procedure rodents with cholinergic parietal lesions showed deficits in attention under conditions of prediction error or surprise.

Briefly, signals that provide partial reinforcement are more likely to prevent new learning, and signals that are consistently reinforced are more likely to form new associations with other signals (Pearce and Hall, 1980). Animals trained on the 5CSRTT with port signals that were either partially or consistently reinforced were then exposed to a pavlovian overshadowing task. Here, the port signals were paired with either a low or high tone. Although PPC ACh-lesioned animals performed normally on the 5CSRTT, they were impaired in the Pavlovian overshadowing task. This was consistent with previous studies showing specific loss of cholinergic input to the parietal cortex resulted in a failure to process conditioned stimuli that predict changes in the value of unconditioned stimuli, an effect interpreted as attention required for new learning (Chiba et al., 1995; Bucci et al., 1998; Maddux et al., 2007). This data has since been interpreted as demonstrating that the ACh reports a mismatch between bottomup stimulus processing and top-down biasing and updates the contextual framework (Yu and Dayan, 2002, 2005; Bucci, 2009).

In a key experiment, St. Peters and colleagues used the dSAT to demonstrate the role of BFCS input to PPC processing (St. Peters et al., 2011). In that experiment, infusions of NMDA into the nucleus accumbens have no effect on standard SAT performance, but improve performance in the dSAT. Further, it was shown that cholinergic deafferentation of either PFC or PPC eliminated the performance-enhancing effects of intra-accumbal NMDA. Thus, this supports the hypothesis that cholinergic transmission in both the PFC and PPC is necessary for attentional effort required to 
overcome challenging conditions, such as the presentation of distractors or compound signals (Sarter et al., 2006). The striatal component of this circuit is crucial for reporting performance errors, and has been discussed in detail elsewhere (Robbins and Everitt, 1996; Sarter et al., 2006).

Neurophysiological studies of the PPC in task performing rats provide evidence of how BFCS input modulates PPC neuronal activity. The presentation of relevant visual signals produces neurophysiological correlates of attention in rodent PPC. Rats were trained on a variant of the SAT and single unit and population activity from the PPC neurons are significantly activated by visual signals. Importantly, neurons were not activated on blank trials and missed signals, and only a small population of neurons was slightly activated by a visual distractor (Broussard et al., 2006). Further, when we recorded the local field potential in the PPC, a prominent P300 evoked response was found when relevant visual signals were presented and subsequently detected (Broussard and Givens, 2010; see below for more detail). Because the P300 is an event-related potential found on parietal sites in humans, this was further neurophysiological evidence of a rodent homolog of the PPC.

\section{PPC RANKS ALLOCENTRIC SIGNALS IN A TEMPORALLY DYNAMIC MANNER TO GUIDE NAVIGATION}

The PPC is also implicated in using external signals to navigate through space. There are two essential strategies a subject can follow for navigation. One can use the nearest landmarks available to determine the route and travel one landmark at a time. Portuguese sailors would travel along the coasts of Africa and Asia using this strategy. A subject can also use distant signals such as the sun and stars to judge their relative position. When the Portuguese sailed to Brazil, they relied on this strategy in the open seas. In psychological terms the use of local signals is called an egocentric strategy whereas the use of distant signals is called allocentric.

One test of egocentric navigation is an eight arm radial maze, where the experimenter places the subject in one arm and reward navigation only to adjacent arms. Here an egocentric strategy requires the subject to go to the nearest arm relative to the subject's initial position in the maze. If the subject begins each daily session in a different arm, it is difficult to use external signals to solve the maze. In this task, parietal lesions have no effect on performance (King and Corwin, 1992). One test of allocentric maze navigation is a cheeseboard task, requiring rodents to learn the position of a food reward on a large table with several recessed food wells. Here, the use of external signals is required to solve the maze. Rats with bilateral PPC lesions took longer paths and had more heading errors (i.e., they started in the wrong direction) than controls (King and Corwin, 1992). In rodents, egocentric signals are presented within a T-maze near the floor whereas allocentric signals are presented on curtains or walls outside of a maze. In rodents unilateral and especially bilateral PPC lesions produced deficits in allocentric navigation, while egocentric navigation remained intact (King and Corwin, 1992; McDaniel et al., 1995, 1998).

Studies investigating the neurophysiological correlates of navigation indicate that as a rodent travels through a path the navigational context dictates the firing pattern of PPC neurons (Chen et al., 1994a,b; Nitz, 2006). For example, if a rat learns to travel a specific route and reverses that route, then the firing pattern on the initial route is very different than the return route. In essence it could mean the temporal order of allocentric signals may influence how PPC ranks the relevance of that cue as the subject navigates through space. Deficits in navigation caused by parietal lesions may be a function of a more basic deficit in the ability of subjects to rank the relevance of external signals in the environment. Although this specific hypothesis has yet to be tested, the medial parietal cortex of human subjects is activated in a virtual reality maze when subjects travel novel, but not familiar, routes (Baumann and Mattingley, 2010). Thus, these basic attentional deficits may also translate into impairment in the ability to remember topological schemas using allocentric signals, a topic covered elsewhere (Goodrich-Hunsaker et al., 2008).

\section{THE NEURAL CIRCUITRY INFLUENCING PARIETAL PROCESSING OF RELEVANT SIGNALS PREFRONTAL-PARIETAL INTERACTIONS IN ATTENTION}

Tasks requiring the filtering of distractors activate both PPC and frontal areas (Hazeltine et al., 2000; Marois et al., 2000; Lee et al., 2006). The activation of these two regions has been dissociated with progressive increases in attentional demand. Bunge and colleagues used a flanking distractor task, where congruent distractors flanking the target aided target detection, and incongruent distractors directed the subject to respond in an opposite manner relative to the target (2002). PPC areas were activated when both congruent and incongruent distractors were presented. Frontal cortex was only significantly activated on trials with incongruent distractors. The distinction here is important, and indicates that PPC actively processes spatial stimuli, and only those distractors that produce response conflicts (incongruent distractors misdirect subjects opposite the correct response) recruit PFC activation.

There is also neurophysiological evidence that PFC is involved in the suppression of distractors. Patients with PFC lesions have exaggerated evoked responses to irrelevant somatosensory and auditory stimuli (Yamaguchi and Knight, 1990). This effect was not replicated in patients with PPC lesions, and controls from this study indicate that the PFC directly suppresses sensory evoked responses. In contrast to this, Friedman-Hill and colleagues demonstrate that a patient with bilateral parietal lesions is impaired when required to filter out perceptually similar distractors, suggesting that the PPC does exhibit top-down selection of relevant visual signals (Friedman-Hill et al., 2003). Thus, the function of the PPC may not be to directly suppress the representation of distractors in sensory cortex, but to disengage from distractors when relevant signals are present (Posner and Petersen, 1990; Posner and Raichle, 1994, Chap. 7). The inability to dynamically rank competing stimuli may result in the impaired selection of relevant stimuli seen in these patients.

Nelson and colleagues (Nelson et al., 2005) investigated some of the mechanisms employed by PFC to modulate PPC activity. In this study perfusion of AMPA and the non-specific ACh agonist carbachol into the PFC increased ACh efflux distally in the PPC. Perfusion of nicotine and NMDA into the PFC did 
not increase PPC ACh efflux, suggesting that muscarinic receptors in the PFC are necessary for PFC to elicit PPC ACh efflux. Perfusion of carbachol or nicotine throughout the PPC, while eliciting increases in local ACh efflux, failed to modulate PFC ACh levels. Moreover, local administration of AMPA into the PPC failed to elicit ACh efflux. These findings suggest PFC input to the BFCS can directly regulate parietal ACh levels. Importantly, PFC also directly projects to the locus coeruleus (LC) (Jodo et al., 1998), suggesting that PFC can modulate cortical levels of both $\mathrm{NE}$ and ACh. Thus, it can be proposed that distractors induce increases in prefrontal ACh that can subsequently recruit parietal ACh efflux in order to differentiate relevant and irrelevant signals (see Figure 1).

\section{CHOLINERGIC MECHANISMS MEDIATING PARIETAL SIGNAL-TO-NOISE RATIO}

Cholinergic neurotransmission has been shown to contribute to visuospatial attention in many paradigms (see earlier, also Botly and De Rosa, 2008). In the SAT, PPC neurons produced neurophysiological correlates of detection, and the hypothesis that cholinergic neurotransmission modulated parietal processing of signals was tested by locally infusing a selective cholinotoxin into the PPC of SAT-performing rats (Broussard et al., 2009). After collecting task-related neurophysiological control data, 192 IgG saporin was infused to deafferent cholinergic neurons projecting locally to PPC (Holley et al., 1994; Wenk et al., 1994). Importantly these were unilateral infusions in order to minimize possible confounding effects on performance.

In standard SAT conditions, PPC neurons from cholinergically deafferented animals are successfully recruited by the visual signal. However, cholinergically deafferention of PPC significantly more neurons responded to the distractor and significantly fewer responded to the signal. Lastly, signal-responsive neurons in deafferented PPC had a lower signal-to-noise ratio (SNR) compared to control conditions and intact subjects. The basal firing rate of these neurons was also elevated during the distractor block of the task. Thus, the cholinergic contribution to PPC processing of signals is hypothesized to provide a basal level of inhibition among local cortical assemblies within the PPC in order to filter distracting stimuli, enhancing the SNR to relevant signals.

As mentioned earlier, the distractor increases the FA rate, producing in several prediction errors that are hypothesized to recruit increased attentional effort. The PFC monitors increases in prediction errors and activates cholinergic signals to normalize the SNR of PPC neurons and filter out task-irrelevant signals (Sarter et al., 2006). The dSAT also elevates PPC cholinergic efflux above

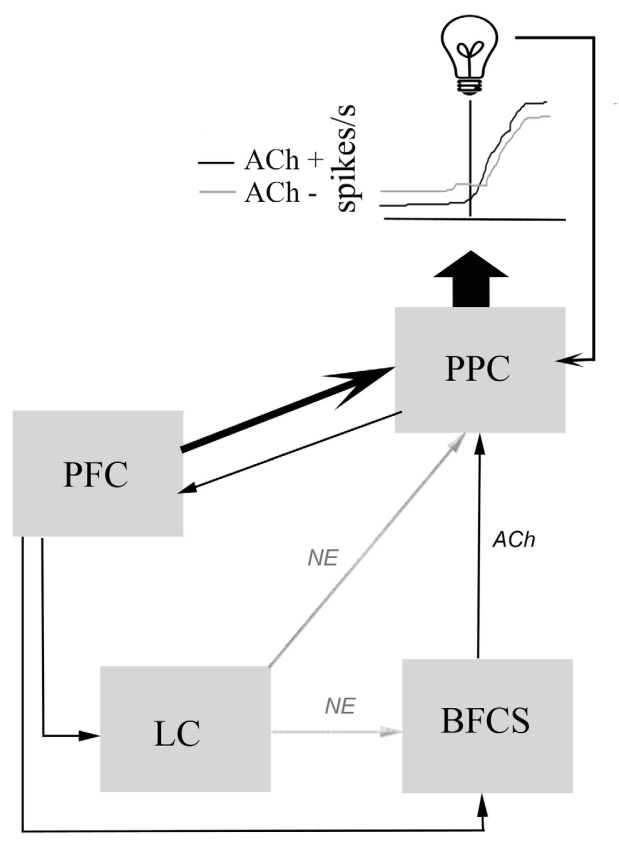

FIGURE 1 | Schematic diagram illustrating the main components of a neuronal network mediating the posterior parietal cortical (PPC) processing of relevant sensory signals. Left, Signals that are well-learned to reliably predict specific outcomes (as represented by the light bulb) require an interaction between frontal, parietal, and sensory cortices to bias the detection and selection of these sensory signals in order to direct proper responses. Neurons in the PPC integrate this input and fire action potentials, represented by the idealized raster plots. Here, neuromodulatory tone from noradrenergic and cholinergic centers only weakly contributes to PPC processing of signals and intact $(\mathrm{ACh}+)$ and cholinergically deafferented subjects (ACh-) have similar responses to relevant signals

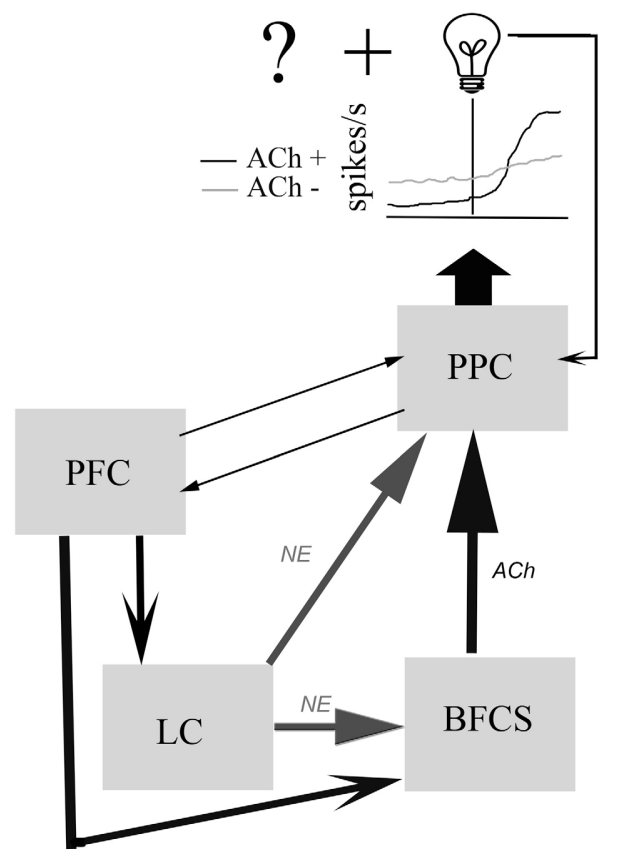

(Broussard et al., 2009). Right, Changes in the ability of learned signals to predict results, either through changes in cue identity, dilution of the signal with intramodal distractors, loss of response contingency, or reversal of response rules will produce prediction errors. In order to overcome these increased errors a motivated subject will require active frontal systems (PFC) to recruit increased cortical neuromodulatory tone (Sarter et al., 2006). The dilution of a visual signal with intramodal distractors unmasks the cholinergic contribution to PPC processing of visual signals. Loss of cholinergic input to the PPC under distractor conditions produces an elevated basal firing rate, and a decreased representation of the visual signal resulting in a reduction in the signal-to-noise ratio. 
normal SAT performance; further evidence of cholinergic influence over PPC processing in the face of challenges requiring attentional effort (Himmelheber et al., 2001; Sarter et al., 2006; St. Peters et al., 2011). This is consistent with the observation in human subjects performing a cued target detection task (Thiel et al., 2005; Giessing et al., 2006; Thiel and Fink, 2008). Here subjects fixate on a central cue that covertly directs them to the right or left visual field. Shortly thereafter, a target appears in either the right or left side and the subject must report detection by pushing a button. Valid cues produce a faster reaction time than invalid cues, a phenomenon known as the "validity effect" (Posner, 1980). Nicotine reduces the validity effect by reducing the reaction time and PPC activity on invalid trials (Thiel et al., 2005; Thiel and Fink, 2008). These effects can be interpreted as an inhibition of the parietal processing of signals with low predictive certainty. Muscarinic neurotransmission is also important as local infusions of scopolamine into the PPC impaired the performance of monkeys on a similar attention task (Davidson et al., 1999).

On a rodent version of the cued target detection task, loss of cortical cholinergic input resulted in decreased accuracy and increased reaction time in response to invalid cues (Bushnell et al., 1998; Chiba et al., 1999). Taken together, this evidence supports the hypothesis that cholinergic input contributes to parietal ranking of the relevance of signals, as increased nicotinic activity discounts processing of low predictive signals and a lack of cholinergic input results in a perseverant processing of invalid signals. Evidence from parietal neurophysiological studies suggests that the cholinergic effects on parietal SNR also influence the validity effect.

The enhancement of the SNR of parietal neurons is consistent with findings of several studies recording evoked responses from brain slices and anesthetized preparations. Application of cholinergic agonists or stimulation of basal forebrain cholinergic nuclei enhanced the cortical responsiveness to sensory stimuli in visual cortex (Sillito and Kemp, 1983; Roberts et al., 2005; Herrero et al., 2008; Roberts and Thiele, 2008; Goard and Dan, 2009), auditory cortex (Metherate et al., 1990), and somatosensory cortex (Alenda and Nunez, 2007). Studies in awake animals demonstrated that the auditory evoked response (Berntson et al., 2003b) of rats to arousal generating stimuli is significantly reduced following loss of cortical cholinergic input. Recordings from brain slices of the anterior cingulate cortex (McCormick and Prince, 1986) demonstrated that exogenous application of ACh produced an initial, phasic hyperpolarization of neurons followed by a tonic depolarization. Focal application of ACh produces a transient inhibition of prefrontal, somatosensory, and visual cortical pyramidal neurons. This effect is produced by activation of M1-like muscarinic receptors. Muscarinic neurotransmission releases calcium from intracellular stores, in turn activating calcium activating potassium channels (SK) (Gulledge and Stuart, 2005; Gulledge et al., 2007). Cortical neurons can inhibit processing from neighboring neurons while enhancing the processing of sensory stimuli within a specific window of time through this general mechanism (Hasselmo and Sarter, 2011).

Current theoretical models regarding the modulation of sensory processing by $\mathrm{ACh}$ are broadened by integrating the contributions of both muscarinic and nicotinic receptor subtypes.
Zinke and colleagues (2006) proposed that ACh reduces lateral cortical integration by acting on M2 receptors that are typically bound presynaptically to local interneurons (Mrzljak et al., 1996; Kimura, 2000). Nicotinic $\alpha 4 \beta 2$ receptors facilitate presynaptic glutamate release from the thalamocortical afferents (Hasselmo and Bower, 1992; Vidal and Changeux, 1993; Gioanni et al., 1999). Although this evidence is derived from primary visual cortex, this could be a general mechanism that ACh employs to produce a shift in cortical processing from local cell assemblies to heightened thalamocortical processing, a shift that may play a role in signal detection (Hasselmo and McGaughy, 2004; Sarter et al., 2005).

A recent study characterized the inhibition of unitary (i.e., one synapse) cortico-cortical connections in the presence of carbachol and nicotinic agonists (Levy et al., 2006). Recordings from somatosensory cortex in vitro indicated that when a layer 5 pyramidal cell was stimulated, neighboring cortical cells $<100 \mu \mathrm{m}$ away generated excitatory post-synaptic potentials (EPSPs). In the presence of non-specific ACh agonist carbachol, the EPSP of cells neighboring the stimulated neuron was reduced, an effect that was blocked by muscarinic antagonists atropine. Further investigation showed that blocking M2 receptors was more effective than blocking M1 receptors in reversing the carbachol-induced suppression. Nicotinic agonists reduced unitary EPSPs only in the absence of $\mathrm{Mg}^{2+}$, suggesting that only in the presence of stimulation significant enough to unblock NMDARs would induce unitary intracortical suppression.

In conclusion, evidence investigating the mechanisms of ACh modulation of cortical responsiveness supports the general hypothesis that ACh increases the signal-related responses of cortical neurons relative to the background firing rate (i.e., the "SNR"). Importantly, in recordings from PPC neurons, cholinergic deafferentation was reduced during distractor sessions, suggesting that cholinergic neurotransmission may facilitate the filtering of distracting stimuli.

\section{NORADRENERGIC MODULATION OF SENSORY PROCESSING}

In addition to the contribution of the cortical cholinergic system to stimulus detection and response selection, a general increase in arousal may be required for a subject to counteract unexpected violations of prior expectations. The LC, the main cortically ascending NE nuclei, projects throughout the cortex, including the PPC (Kobayashi et al., 1974; Descarries et al., 1977), and stimulation of the LC produces NE efflux in both the PPC and PFC (Devoto et al., 2005). NE input to the PPC is dense, and NE input to BF nuclei may contribute to further elevated levels of ACh. The specific contribution of ascending NE inputs to rodent PPC has not been studied, but a prevalence of primate and human studies support the notion that NE contributes to shifts in attention. Neurons in the LC are activated shifts in visual attention and are hypothesized to guide the response late in the decision-making process (Clayton et al., 2004).

In rodents, the dissociation of the roles of cholinergic and NE modulation to attentional processing was exemplified in a study by Dalley and colleagues (Dalley et al., 2001). Here, microdialysis probes implanted in rats revealed that normal 5CSRTT performance elevates cholinergic, but not NE levels in the prefrontal 
cortex. This was consistent from day to day, i.e., the task was well-learned and increases in acetylcholine were resistant to overtraining. In contrast, in rats whose rewards were contingent upon another rats' performance (yoked) increased NE efflux. This effect lasted on the day the contingency was yoked; subsequent sessions did not elevate NE levels. Cortical-wide depletions of NE, but not ACh, impairs performance on attentional set-shifting tasks when the relevant cue shifted dimensions (McGaughy et al., 2008). In addition to this direct neuromodulatory influence on cortical processing, the LC projects to the basal forebrain where it may selectively bias the processing of anxiogenic stimuli (Hart et al., 1999; Berntson et al., 2003a,b). Whether NE contributes to parietal processing of targets in the 5CSRTT or SAT remains an open question.

Based on these and other findings, cholinergic neurotransmission within the cortex is thought to mediate expected uncertainty, i.e., defined as known degree of unreliability of predictive signals within a given environment. NE neurotransmission within the cortex is thought to compensate for unexpected uncertainty, when global changes in cue identity or task rules violate prior expectations (Yu and Dayan, 2005). A recent modeling simulation exemplifies this concept (Avery et al., 2012). In it a subject is placed in a circular field surrounded by 36 lights each $10^{\circ}$ apart. On any trial a light directs a simulated rodent to a port, and the subject is required to break a light beam in the lit port and return to the center for a reward. In this paradigm the experimenter controls the mean and standard deviation of the relevant light. Within a fixed number of trials, the mean (from 0 to $360^{\circ}$ ) and standard deviation remain fixed so that an expected range of relevant lights can be monitored. This reflects the degree of expected uncertainty in the paradigm. After a fixed number of trials the experimenter shifts the mean, violating prior expectations set by previous trials; this represents unexpected uncertainty. In this biologically based model phasic bursts of ACh track small deviations from the mean signal presentation region, whereas bursts of NE track global changes in the mean. As valuable as these modeling data are, there are few studies that investigate the role of NE specifically in PPC, and it is yet to be determined whether NE efflux in the PFC alone is sufficient to overcome enhanced unexpected uncertainty.

Another unresolved issue is that it is yet to be determined whether distractors present expected or unexpected uncertainty. The SAT, as mentioned earlier, elevates cortical ACh (Arnold et al., 2002), i.e., in turn further elevated by distractors (St. Peters et al., 2011). This and other findings have been the basis of the construct attentional effort that require a motivated subject must allocate additional attentional resources to overcome challenges (Sarter et al., 2006). But in this paradigm the distractor does not violate the predictive validity of a cue, but it does dilute the relevant signal with several false signals. It would be interesting to test the hypothesis that distractor-related increases in cortical ACh are a function of elevated NE drive to BFCS [see Figure 3 of Sarter et al. (2006)].

Some of the mechanisms underlying NE influence on cortical processing have been studied. NE neurotransmission via beta receptors modulates the responsiveness of sensory neurons. Specifically, stimulation of rodent whiskers at levels that do not activate barrel cortex is enhanced when the stimulation frequency of the LC is increased. This increase in stimulation frequency represents a change in the firing rate of the $\mathrm{LC}$ and it is proposed that this elevates cortical NE, enhances the sensory processing of subthreshold stimuli (Devilbiss and Waterhouse, 2004).

\section{PARIETAL LOCAL FIELD POTENTIALS IN SAT-PERFORMING ANIMALS}

Extensive reviews focusing on the distinction between unit activity and LFP activity have been published (Logothetis, 2003; Buzsaki, 2006). Single unit recordings measure the extracellular field potential when microelectrodes are placed close to the soma or axons of a neuron, and reports the action potentials produced by the nearest population of neurons. The firing rate of neurons has been a critical measure for comparing the neural activity of sensory processing or behavior for decades (Mountcastle et al., 1975; Boudreau et al., 2006). Measuring single unit activity provides no information about subthreshold inputs to dendritic arbors or integrative processing in the soma.

By contrast, LFPs represents the cooperative activity of neural populations. Rhythmic LFPs of high amplitude and low frequency, classified originally in the EEG literature as delta and theta oscillations, and are generated by the interaction of thalamocortical and neocortical activity and are typically modulated by the ascending neurotransmitter systems, such as ACh, NE, and histamine (Steriade et al., 1993; Eggermann et al., 2001; Lee et al., 2005).

Studies that simultaneously measure single unit and LFP activity demonstrated that the firing rate of single neurons can be gated in part by the oscillations in the local field (Costa et al., 2006). When the local extracellular field is positive, neurons embedded within the field are inhibited; as the intracellular milieu approaches threshold potential the conditions are more favorable to local cell firing. Conversely, as the local extracellular field is negative, it can lower the firing threshold of neurons embedded within that field, thereby temporally constraining the firing of neurons.

There are several measures of the field potential that correlate with either bottom-up processing of salience or top-down biasing of choices. One such measure is the $\mathrm{P} 300$ response, a positive extracellular potential that peaks around $300 \mathrm{~ms}$ post signal in humans. In terms of the classic EEG literature the P300 is a single, high amplitude cycle of the delta oscillation (Polich, 2007), that has a maximal amplitude in humans and primates at parietal sites (Linden, 2005). The standard paradigm used to generate the P300 response is the "oddball task," where infrequent targets are successively presented with frequent targets of varying relevance. The amplitude of the P300 varies as a function of stimulus discriminability, and was also found in rats (Broussard and Givens, 2010). In the SAT, short duration signals and distractors did not produce a P300 response. However, highly salient $500 \mathrm{~ms}$ signals produced a considerable $\mathrm{P} 300$ response from SAT-performing rats.

Another component of the field potential related to task performance is the long-latency (500-1000 ms post signal) contingent negative variation $(\mathrm{CNV})$. The $\mathrm{CNV}$ was first measured from the scalp of humans and has two components. The first component is generated in anterior areas over the frontal eye field 
and is developed after a stimulus calls for a decision. The second component is found over more central areas and is related to the execution of a prepared response plan (Singh et al., 1990). In PPC, the $\mathrm{CNV}$ is more likely to correspond to central generation of the $\mathrm{CNV}$ and reflect correct responses to targets (Le Dantec et al., 2007). In SAT-performing rats recording from PPC LFP revealed a detection-specific CNV. Unlike the P300, the CNV-like response in rat PPC was the same regardless of signal duration (Broussard and Givens, 2010). The CNV correlated with an increasing alpha power and an increase in firing rate of PPC neurons in SATperforming rats. It must be reiterated here that neither $\mathrm{CNV}$-like responses nor phasic increases in firing rate occurred on nonsignal trials, indicating that these events underlie an effortful processing of a signal preceding a response.

Because the P300 is an extracellar positive potential, it is hypothesized that it is a wave of inhibition that sweeps throughout the PPC, minimizing local cortico-cortical activity (Polich, 2007). This ongoing activity may reflect the maintenance of a response plan in working memory. In the SAT task, rats perform better on high-probability on non-signal trials, and SATperforming rats have been shown to position themselves near the non-signal response lever. This evidence supports the hypothesis that rats maintain a non-signal response plan in working memory during the intertrial interval. Phasic cholinergic signaling here may also contribute to facilitate nicotinic currents influencing thalamocortical circuitry, resulting in increased firing rate of PPC neurons prior to proper responding on signal trials. Muscarinic receptor neurotransmission acting on a slower timescale (seconds to minutes) can lower the baseline firing rate on subsequent trials. Muscarinic and NE signaling acting at this timescale could also potentiate the P300 response, phasically inhibiting local parietal cell assemblies and facilitating a shift to thalamocortical processing. Then, the extracellularly negative $\mathrm{CNV}$ potential could act to disinhibit PPC activity to maintain sensory-driven neuronal spiking generated by specific PPC neurons.

\section{REFERENCES}

Alenda, A., and Nunez, A. (2007). Cholinergic modulation of sensory interference in rat primary somatosensory cortical neurons. Brain Res. 1133, 158-167.

Andersen, R. A., and Buneo, C. A. (2002). Intentional maps in posterior parietal cortex. Annu. Rev. Neurosci. 25, 189-220.

Arnold, H. M., Burk, J. A., Hodgson, E. M., Sarter, M., and Bruno, J. P. (2002). Differential cortical acetylcholine release in rats performing a sustained attention task versus behavioral control tasks that do not explicitly tax attention. Neuroscience 114, 451-460.

Avery, M. C., Nitz, D. A., Chiba, A. A., and Krichmar, J. L. (2012). Simulation of cholinergic and noradrenergic modulation of behavior in uncertain environments. Front.

\section{WORKING MODEL/CONCLUSIONS}

To summarize, the PPC is hypothesized to be a necessary component of an attentional network comprised of PFC, thalamus, striatum, and neuromodulatory influence from ascending cholinergic and NE nuclei. Parietal neurons fire in response to behaviorally relevant stimuli but only when subjects report that they have detected them (i.e., not on miss trials). When attentional demands and uncertainty levels are static, neuromodulatory influence may not be necessary for parietal processing of signals. However, presenting distractors, novel compound stimuli, or other task manipulations requiring new learning recruit's increases in neuromodulatory tone to dynamically update associations within the PPC by modifying the firing rate and thus the ranking of these signals. Cholinergic input may be recruited in conditions of elevated expected uncertainty, such as when a subject must monitor and consciously ignore known distractors and attend to behaviorally relevant signals. Cholinergic signaling may act through nicotinic receptors at rapid timescales to facilitate thalamocortical processing and muscarinic receptors at slower timescales to inhibit local recurrent cell assemblies, in doing so lower the basal firing rate of parietal neurons and enhance the SNR of their response to relevant signals. The contribution of NE input to parietal attentional processing is less clear, but models suggest that NE efflux facilitates inhibition within the cortex, contributing for example to the globally inhibiting P300 response. Also, projections from LC to the BF serve to further elevate cortical ACh under conditions requiring additional attentional effort. The inhibition facilitated by the influence of NE and ACh on local circuitry within the PPC may act to clear the contents of working memory and bias parietal neurons in favor of processing incoming signals so as to generate the optimal behavioral response.

\section{ACKNOWLEDGMENTS}

The author would like to thank Kechun Yang and Alyse Thomas for critical reading of earlier drafts of the manuscript.

by cortical cholinergic deafferentation. Neuroscience 116, 179-186.

Bisley, J. W., and Goldberg, M. E. (2006). Neural correlates of attention and distractibility in the lateral intraparietal area. J. Neurophysiol. 95, 1696-1717.

Bisley, J. W., Krishna, B. S., and Goldberg, M. E. (2004). A rapid and precise on-response in posterior parietal cortex. J. Neurosci. 24, 1833-1838.

Botly, L. C., and De Rosa, E. (2008). A cross-species investigation of acetylcholine, attention, and feature binding. Psychol. Sci. 19, 1185-1193.

Boudreau, C. E., Williford, T. H., and Maunsell, J. H. (2006). Effects of task difficulty and target likelihood in area V4 of macaque monkeys. J. Neurophysiol. 96, 2377-2387.
Broussard, J., Sarter, M., and Givens, B. (2006). Neuronal correlates of signal detection in the posterior parietal cortex of rats performing a sustained attention task. Neuroscience 143, 407-417.

Broussard, J. I., and Givens, B. (2010). Low frequency oscillations in rat posterior parietal cortex are differentially activated by cues and distractors. Neurobiol. Learn. Mem. 94, 191-198.

Broussard, J. I., Karelina, K., Sarter, M., and Givens, B. (2009). Cholinergic optimization of cue-evoked parietal activity during challenged attentional performance. Eur. J. Neurosci. 29, 1711-1722.

Bucci, D. J. (2009). Posterior parietal cortex: an interface between attention and learning? Neurobiol. Learn. Mem. 91, 114-120. 
Bucci, D. J., Conley, M., and Gallagher, M. (1999). Thalamic and basal forebrain cholinergic connections of the rat posterior parietal cortex. Neuroreport 10, 941-945.

Bucci, D. J., Holland, P. C., and Gallagher, M. (1998). Removal of cholinergic input to rat posterior parietal cortex disrupts incremental processing of conditioned stimuli. J. Neurosci. 18, 8038-8046.

Bushnell, P. J., Chiba, A. A., and Oshiro, W. M. (1998). Effects of unilateral removal of basal forebrain cholinergic neurons on cued target detection in rats. Behav. Brain Res. 90, 57-71.

Bushnell, P. J., Kelly, K. L., and Crofton, K. M. (1994). Effects of toluene inhalation on detection of auditory signals in rats. Neurotoxicol. Teratol. $16,149-160$.

Buzsaki, G. (2006). Rhythms of the Brain, 1st Edn. New York, NY: Oxford University Press.

Carli, M., Robbins, T. W., Evenden, J. L., and Everitt, B. J. (1983). Effects of lesions to ascending noradrenergic neurones on performance of a 5-choice serial reaction task in rats; implications for theories of dorsal noradrenergic bundle function based on selective attention and arousal. Behav. Brain Res. 9, 361-380.

Chandler, H. C., King, V., Corwin, J. V., and Reep, R. L. (1992). Thalamocortical connections of rat posterior parietal cortex. Neurosci. Lett. 143, 237-242.

Chen, L. L., Lin, L. H., Barnes, C. A., and McNaughton, B. L. (1994a). Head-direction cells in the rat posterior cortex. II. Contributions of visual and ideothetic information to the directional firing. Exp. Brain Res. 101, 24-34.

Chen, L. L., Lin, L. H., Green, E. J., Barnes, C. A., and McNaughton, B. L. (1994b). Head-direction cells in the rat posterior cortex. I. Anatomical distribution and behavioral modulation. Exp. Brain Res. 101, 8-23.

Chiba, A. A., Bucci, D. J., Holland, P. C., and Gallagher, M. (1995). Basal forebrain cholinergic lesions disrupt increments but not decrements in conditioned stimulus processing. J. Neurosci. 15, 7315-7322.

Chiba, A. A., Bushnell, P. J., Oshiro, W. M., and Gallagher, M. (1999). Selective removal of cholinergic neurons in the basal forebrain alters cued target detection. Neuroreport 10, 3119-3123.

Clayton, E. C., Rajkowski, J., Cohen, J. D., and Aston-Jones, G. (2004). Phasic activation of monkey locus ceruleus neurons by simple decisions in a forced-choice task. $J$. Neurosci. 24, 9914-9920.

Cohen, Y. E., and Andersen, R. A. (2002). A common reference frame for movement plans in the posterior parietal cortex. Nat. Rev. Neurosci. 3 , 553-562.

Colby, C. L., and Goldberg, M. E. (1999). Space and attention in parietal cortex. Annu. Rev. Neurosci. 22, 319-349.

Costa, R. M., Lin, S. C., Sotnikova, T. D., Cyr, M., Gainetdinov, R. R., Caron, M. G., and Nicolelis, M. A. (2006). Rapid alterations in corticostriatal ensemble coordination during acute dopamine-dependent motor dysfunction. Neuron 52, 359-369.

Critchley, M. (1953). The Parietal Lobes. New York, NY: Hafner publishing company.

Dalley, J. W., McGaughy, J., O'Connell, M. T., Cardinal, R. N., Levita, L., and Robbins, T. W. (2001). Distinct changes in cortical acetylcholine and noradrenaline efflux during contingent and noncontingent performance of a visual attentional task. J. Neurosci. 21, 4908-4914.

Davidson, M. C., Cutrell, E. B., and Marrocco, R. T. (1999). Scopolamine slows the orienting of attention in primates to cued visual targets. Psychopharmacology 142, $1-8$.

Descarries, L., Watkins, K. C., and Lapierre, Y. (1977). Noradrenergic axon terminals in the cerebral cortex of rat. III. Topometric ultrastructural analysis. Brain Res. 133, 197-222.

Devilbiss, D. M., and Waterhouse, B. D. (2004). The effects of tonic locus ceruleus output on sensoryevoked responses of ventral posterior medial thalamic and barrel field cortical neurons in the awake rat. J. Neurosci. 24, 10773-10785.

Devoto, P., Flore, G., Saba, P., Fa, M., and Gessa, G. L. (2005). Stimulation of the locus coeruleus elicits noradrenaline and dopamine release in the medial prefrontal and parietal cortex. J. Neurochem. 92, 368-374.

Eggermann, E., Serafin, M., Bayer, L., Machard, D., Saint-Mleux, B., Jones, B. E., and Muhlethaler, M. (2001). Orexins/hypocretins excite basal forebrain cholinergic neurones. Neuroscience 108, 177-181.

Friedman-Hill, S. R., Robertson, L. C., Desimone, R., and Ungerleider, L. G. (2003). Posterior parietal cortex and the filtering of distractors. Proc. Natl. Acad. Sci. U.S.A. 100, 4263-4268.
Giessing, C., Thiel, C. M., Rosler, F., and Fink, G. R. (2006). The modulatory effects of nicotine on parietal cortex activity in a cued target detection task depend on cue reliability. Neuroscience 137, 853-864.

Gill, T. M., Sarter, M., and Givens, B. (2000). Sustained visual attention performance-associated prefrontal neuronal activity: evidence for cholinergic modulation. J. Neurosci. 20, 4745-4757.

Gioanni, Y., Rougeot, C., Clarke, P. B., Lepouse, C., Thierry, A. M., and Vidal, C. (1999). Nicotinic receptors in the rat prefrontal cortex: increase in glutamate release and facilitation of mediodorsal thalamo-cortical transmission. Eur. J. Neurosci. 11, $18-30$.

Goard, M., and Dan, Y. (2009). Basal forebrain activation enhances cortical coding of natural scenes. Nat. Neurosci. 12, 1444-1449.

Goodrich-Hunsaker, N. J., Howard, B. P., Hunsaker, M. R., and Kesner, R. P. (2008). Human topological task adapted for rats: spatial information processes of the parietal cortex. Neurobiol. Learn. Mem. 90, 389-394.

Gulledge, A. T., Park, S. B., Kawaguchi, Y., and Stuart, G. J. (2007). Heterogeneity of phasic cholinergic signaling in neocortical neurons. J. Neurophysiol. 97, 2215-2229.

Gulledge, A. T., and Stuart, G. J. (2005). Cholinergic inhibition of neocortical pyramidal neurons. J. Neurosci. 25, 10308-10320.

Hart, S., Sarter, M., and Berntson, G. G. (1999). Cholinergic inputs to the rat medial prefrontal cortex mediate potentiation of the cardiovascular defensive response by the anxiogenic benzodiazephine receptor partial inverse agonist FG (7142). Neuroscience 94, 1029-1038.

Hasselmo, M. E., and Bower, J. M. (1992). Cholinergic suppression specific to intrinsic not afferent fiber synapses in rat piriform (olfactory) cortex. J. Neurophysiol. 67, 1222-1229.

Hasselmo, M. E., and McGaughy, J. (2004). High acetylcholine levels set circuit dynamics for attention and encoding and low acetylcholine levels set dynamics for consolidation. Prog. Brain Res. 145, 207-231.

Hasselmo, M. E., and Sarter, M. (2011). Modes and models of forebrain cholinergic neuromodulation of cognition. Neuropsychopharmacology 36 , 52-73.

Hazeltine, E., Poldrack, R., and Gabrieli, J. D. (2000). Neural activation during response competition.
J. Cogn. Neurosci. 12(Suppl. 2), 118-129.

Herrero, J. L., Roberts, M. J., Delicato, L. S., Gieselmann, M. A., Dayan, P., and Thiele, A. (2008). Acetylcholine contributes through muscarinic receptors to attentional modulation in V1. Nature 454 1110-1114.

Himmelheber, A. M., Sarter, M., and Bruno, J. P. (2001). The effects of manipulations of attentional demand on cortical acetylcholine release. Brain Res. Cogn. Brain Res. 12, 353-370.

Holley, L. A., Wiley, R. G., Lappi, D. A., and Sarter, M. (1994). Cortical cholinergic deafferentation following the intracortical infusion of 192 IgG-saporin: a quantitative histochemical study. Brain Res. 663, 277-286.

Ipata, A. E., Gee, A. L., Gottlieb, J., Bisley, J. W., and Goldberg, M. E. (2006). LIP responses to a popout stimulus are reduced if it is overtly ignored. Nat. Neurosci. 9, 1071-1076

Jodo, E., Chiang, C., and AstonJones, G. (1998). Potent excitatory influence of prefrontal cortex activity on noradrenergic locus coeruleus neurons. Neuroscience 83, 63-79.

Kalaska, J. F. (1996). Parietal cortex area 5 and visuomotor behavior. Can. J. Physiol. Pharmacol. 74, 483-498.

Kastner, S., and Ungerleider, L. G. (2000). Mechanisms of visual attention in the human cortex. Ann. Rev. Neurosci. 23, 315-341.

Kimura, F. (2000). Cholinergic modulation of cortical function: a hypothetical role in shifting the dynamics in cortical network. Neurosci. Res. $38,19-26$.

King, V. R., and Corwin, J. V. (1992) Spatial deficits and hemispheric asymmetries in the rat following unilateral and bilateral lesions of posterior parietal or medial agranular cortex. Behav. Brain Res. 50, 53-68.

King, V. R., and Corwin, J. V. (1993). Comparisons of hemi-inattention produced by unilateral lesions of the posterior parietal cortex or medial agranular prefrontal cortex in rats: neglect, extinction, and the role of stimulus distance. Behav. Brain Res. 54, 117-131.

Kobayashi, R. M., Palkovits, M., Kopin, I. J., and Jacobowitz, D. M. (1974). Biochemical mapping of noradrenergic nerves arising from the rat locus coeruleus. Brain Res. 77, 269-279.

Le Dantec, C., Gontier, E., Paul, I., Charvin, H., Bernard, C., Lalonde, 
R., and Rebai, M. (2007). ERPs associated with visual duration discriminations in prefrontal and parietal cortex. Acta Psychol. (Amst.) 125, $85-98$

Lee, K. H., Choi, Y. Y., Gray, J. R., Cho, S. H., Chae, J. H., Lee, S., and Kim, K. (2006). Neural correlates of superior intelligence: stronger recruitment of posterior parietal cortex. Neuroimage 29, 578-586.

Lee, M. G., Hassani, O. K., Alonso, A., and Jones, B. E. (2005). Cholinergic basal forebrain neurons burst with theta during waking and paradoxical sleep. J. Neurosci. 25, 4365-4369.

Leichnetz, G. R. (2001). Connections of the medial posterior parietal cortex (area $7 \mathrm{~m}$ ) in the monkey. Anat. Rec. 263, 215-236.

Levy, R. B., Reyes, A. D., and Aoki, C. (2006). Nicotinic and muscarinic reduction of unitary excitatory postsynaptic potentials in sensory cortex; dual intracellular recording in vitro. J. Neurophysiol. 95, 2155-2166.

Linden, D. E. (2005). The p300, where in the brain is it produced and what does it tell us? Neuroscientist 11, 563-576.

Logothetis, N. K. (2003). The underpinnings of the BOLD functional magnetic resonance imaging signal. J. Neurosci. 23, 3963-3971.

Maddux, J. M., Kerfoot, E. C., Chatterjee, S., and Holland, P. C. (2007). Dissociation of attention in learning and action: effects of lesions of the amygdala central nucleus, medial prefrontal cortex, and posterior parietal cortex. Behav. Neurosci. 121, 63-79.

Marois, R., Chun, M. M., and Gore, J. C. (2000). Neural correlates of the attentional blink. Neuron 28, 299-308.

McCormick, D. A., and Prince, D. A. (1986). Mechanisms of action of acetylcholine in the guinea-pig cerebral cortex in vitro. J. Physiol. 375, 169-194.

McDaniel, W. F., Via, J. D., Smith, J. S., Wells, D. L., Fu, J. J., Bishop, J. F., Boyd, P. A., and Ledesma, H. M. (1995). Unilateral injury of posterior parietal cortex and spatial learning in hooded rats. Behav. Brain Res. 70, 165-179.

McDaniel, W. F., Williams, L. B., Cullen, M. A., and Compton, D. M. (1998). Turn-signal utilization by rats with either unilateral or bilateral posterior parietal cortex injuries. Psychobiology 26, 143-152.
McGaughy, J., Kaiser, T., and Sarter, M. (1996). Behavioral vigilance following infusions of $192 \mathrm{IgG}$-saporin into the basal forebrain: selectivity of the behavioral impairment and relation to cortical AChE-positive fiber density. Behav. Neurosci. 110, 247-265.

McGaughy, J., Ross, R. S., and Eichenbaum, H. (2008). Noradrenergic, but not cholinergic, deafferentation of prefrontal cortex impairs attentional set-shifting. Neuroscience 153, 63-71.

McGaughy, J., and Sarter, M. (1995). Behavioral vigilance in rats: task validation and effects of age, amphetamine and benzodiazepine receptor ligands. Psychopharmacology 117, 340-357.

Metherate, R., Ashe, J. H., and Weinberger, N. M. (1990). Acetylcholine modifies neuronal acoustic rate-level functions in guinea pig auditory cortex by an action at muscarinic receptors. Synapse 6, 364-368.

Mountcastle, V. B., Lynch, J. C., Georgopoulos, A., Sakata, H., and Acuna, C. (1975). Posterior parietal association cortex of the monkey: command functions for operations within extrapersonal space. J. Neurophysiol. 38, 871-908.

Mrzljak, L., Levey, A. I., and Rakic, P. (1996). Selective expression of $\mathrm{m} 2$ muscarinic receptor in the parvocellular channel of the primate visual cortex. Proc. Natl. Acad. Sci. U.S.A. 93, 7337-7340.

Muir, J. L., Everitt, B. J., and Robbins, T. W. (1994). AMPA-induced excitotoxic lesions of the basal forebrain: a significant role for the cortical cholinergic system in attentional function. J. Neurosci. 14, 2313-2326.

Nelson, C., Sarter, M., and Bruno, J. (2005). Prefrontal cortical modulation of acetylcholine release in posterior parietal cortex. Neuroscience $132,347-359$

Nitz, D. A. (2006). Tracking route progression in the posterior parietal cortex. Neuron 49, 747-756.

Pare, M., and Wurtz, R. H. (1997). Monkey posterior parietal cortex neurons antidromically activated from superior colliculus. $J$. Neurophysiol. 78, 3493-3497.

Pearce, J. M., and Hall, G. (1980). A model for Pavlovian learning: variations in the effectiveness of conditioned but not of unconditioned stimuli. Psychol. Rev. 87, 532-552.
Polich, J. (2007). Updating P300, an integrative theory of $\mathrm{P} 3 \mathrm{a}$ and $\mathrm{P} 3 \mathrm{~b}$. Clin. Neurophysiol. 118, 2128-2148.

Posner, M. I. (1980). Orienting of attention. Q. J. Exp. Psychol. 32, 3-25.

Posner, M. I., and Petersen, S. E. (1990). The attention system of the human brain. Annu. Rev. Neurosci. 13, 25-42.

Posner, M. I., and Raichle, M. E. (1994). Images of Mind. New York, NY W. H. Freeman.

Posner, M. I., Snyder, C., and Davidson, B. (1980). Attention and detection of signals. J. Exp. Psychol. 109, 160-174.

Reep, R. L., Chandler, H. C., King, V., and Corwin, J. V. (1994). Rat posterior parietal cortex: topography of corticocortical and thalamic connections. Exp. Brain Res. 100, 67-84.

Reep, R. L., and Corwin, J. V. (2009). Posterior parietal cortex as part of a neural network for directed attention in rats. Neurobiol. Learn. Mem. 91, 104-113.

Robbins, T. W., and Everitt, B. J. (1996). Neurobehavioural mechanisms of reward and motivation. Curr. Opin. Neurobiol. 6, 228-236.

Roberts, M. J., and Thiele, A. (2008). Spatial integration and its moderation by attention and acetylcholine. Front. Biosci. 13, 3742-3759.

Roberts, M. J., Zinke, W., Guo, K., Robertson, R., McDonald, J. S., and Thiele, A. (2005). Acetylcholine dynamically controls spatial integration in marmoset primary visual cortex. J. Neurophysiol. 93, 2062-2072.

Rushworth, M. F., and Taylor, P. C. (2006). TMS in the parietal cortex: updating representations for attention and action. Neuropsychologia 44, 2700-2716.

Sarter, M., Gehring, W. J., and Kozak, R. (2006). More attention must be paid: the neurobiology of attentional effort. Brain Res. Rev. 51, 145-160.

Sarter, M., Hasselmo, M., Bruno, J. P. and Givens, B. (2005). Unraveling the attentional functions of cortical cholinergic inputs: interactions between signal-driven and cognitive modulation of signal detection. Brain Res. Rev. 48, 98-111.

Scherberger, H., Jarvis, M. R., and Andersen, R. A. (2005). Cortical local field potential encodes movement intentions in the posterior parietal cortex. Neuron 46, 347-354.

Schultz, W., and Dickinson, A. (2000). Neuronal coding of prediction errors. Annu. Rev. Neurosci. 23, 473-500.

Sereno, M. I., Pitzalis, S., and Martinez, A. (2001). Mapping of contralateral space in retinotopic coordinates by a parietal cortical area in humans. Science 294, 1350-1354.

Sillito, A. M., and Kemp, J. A. (1983). Cholinergic modulation of the functional organization of the cat visual cortex. Brain Res. 289, 143-155.

Singh, J., Knight, R. T., Woods, D. L., Beckley, D. J., and Clayworth, C. (1990). Lack of age effects on human brain potentials preceding voluntary movements. Neurosci. Lett. 119, 27-31.

Snyder, L. (2000). Moving forward by looking away. Nature 408, 921-923.

St. Peters, M., Demeter, E., Lustig, C., Bruno, J. P., and Sarter, M. (2011). Enhanced control of attention by stimulating mesolimbiccorticopetal cholinergic circuitry. J. Neurosci. 31, 9760-9771.

Steriade, M., McCormick, D. A., and Sejnowski, T. J. (1993). Thalamocortical oscillations in the sleeping and aroused brain. Science 262, 679-685.

Thiel, C. M., and Fink, G. R. (2008). Effects of the cholinergic agonist nicotine on reorienting of visual spatial attention and top-down attentional control. Neuroscience 152, 381-390.

Thiel, C. M., Zilles, K., and Fink, G. R. (2005). Nicotine modulates reorienting of visuospatial attention and neural activity in human parietal cortex. Neuropsychopharmacology 30, 810-820.

Vanduffel, W., Fize, D., Mandeville, J. B., Nelissen, K., Van Hecke, P., Rosen, B. R., Tootell, R. B., and Orban, G. A. (2001). Visual motion processing investigated using contrast agent-enhanced fMRI in awake behaving monkeys. Neuron 32, 565-577.

Vanduffel, W., Fize, D., Peuskens, H., Denys, K., Sunaert, S., Todd, J. T., and Orban, G. A. (2002) Extracting 3D from motion: differences in human and monkey intraparietal cortex. Science 298, 413-415.

Vidal, C., and Changeux, J. P. (1993). Nicotinic and muscarinic modulations of excitatory synaptic transmission in the rat prefrontal cortex in vitro. Neuroscience $56,23-32$.

Wenk, G. L., Stoehr, J. D., Quintana, G., Mobley, S., and Wiley, R. G. (1994). Behavioral, biochemical, histological, and electrophysiological effects of 192 IgG-saporin injections 
into the basal forebrain of rats. J. Neurosci. 14, 5986-5995.

Yamaguchi, S., and Knight, R. T. (1990). Gating of somatosensory input by human prefrontal cortex. Brain Res. 521, 281-288.

Yu, A. J., and Dayan, P. (2002). Acetylcholine in cortical inference. Neural Netw. 15, 719-730.

Yu, A. J., and Dayan, P. (2005). Uncertainty, neuromodulation, and attention. Neuron 46, 681-692.
Zinke, W., Roberts, M. J., Guo, K., McDonald, J. S., Robertson, R., and Thiele, A. (2006). Cholinergic modulation of response properties and orientation tuning of neurons in primary visual cortex of anaesthetized Marmoset monkeys. Eur. J. Neurosci. 24, 314-328.

Conflict of Interest Statement: The author declares that the research was conducted in the absence of any commercial or financial relationships that could be construed as a potential conflict of interest.

Received: 24 March 2012; paper pending published: 30 April 2012; accepted: 28 May 2012; published online: 14 June 2012

Citation: Broussard IJ (2012) Posterior parietal cortex dynamically ranks topographic signals via cholinergic influence. Front. Integr. Neurosci. 6:32. doi: 10.3389/fnint.2012.00032

Copyright (c) 2012 Broussard. This is an open-access article distributed under the terms of the Creative Commons Attribution Non Commercial License, which permits non-commercial use, distribution, and reproduction in other forums, provided the original authors and source are credited. 